\title{
Infertility evaluation and access to assisted reproductive technologies among male military veterans: analysis of the South Florida Veterans Affairs experience
}

\author{
Joshua A. Halpern ${ }^{1}$, Joshua S. Jue ${ }^{2}$, Ranjith Ramasamy ${ }^{2}$ \\ ${ }^{1}$ Department of Urology, New York Presbyterian Hospital, Weill Cornell Medicine, New York, NY, USA; ${ }^{2}$ Department of Urology, University of \\ Miami Miller School of Medicine, Miami, FL, USA \\ Contributions: (I) Conception and design: All authors; (II) Administrative support: R Ramasamy; (III) Provision of study material or patients: R \\ Ramasamy; (IV) Collection and assembly of data: R Ramasamy, JS Jue; (V) Data analysis and interpretation: All authors; (VI) Manuscript writing: All \\ authors; (VII) Final approval of manuscript: All authors. \\ Correspondence to: Ranjith Ramasamy. Department of Urology, University of Miami Miller School of Medicine, Miami, FL, USA. \\ Email: Ramasamy@miami.edu.
}

\begin{abstract}
Background: Recent legislation extended coverage for assisted reproductive technologies (ART) to male veterans within the United States Veterans Affairs (VA) healthcare system. We sought to characterize the appropriateness of male infertility diagnosis and access to assisted reproductive technologies (ART) among men within the local VA healthcare system after passage of the new legislation.

Methods: We retrospectively identified male patients within the South Florida VA Network who underwent semen analysis (SA) or were diagnosed with infertility between January 1999 and June 2017. Men were classified by infertility diagnosis and sperm concentration/mL (normal, oligospermia $<15$, severe oligospermia $<5$ million, azoospermia). We compared the number of oligospermic men before versus after the legislation. Men who did not receive ART were characterized according to eligibility criteria of military service connection to the infertility diagnosis.
\end{abstract}

Results: Six hundred twenty-seven men underwent SA or were diagnosed with infertility. Among 474 men with SA, 206 (43.5\%) were diagnosed with infertility and 268 (56.5\%) were not. Additionally, 153 men received an infertility diagnosis without SA. More men had oligospermia and severe oligospermia after the legislation (1 vs. 6, 1 vs. 10). Of 10 men with severe oligospermia post-legislation, 7 did not proceed to ART consultation due to lack of military service connection to the infertility diagnosis.

Conclusions: A substantial proportion of veterans with abnormal sperm concentrations were not diagnosed with infertility, while others carrying an infertility diagnosis did not undergo SA. Most patients clinically eligible for ART did not proceed with further evaluation due to lack of military service connection to their infertility diagnosis.

Keywords: Veterans; infertility; male; assisted reproductive techniques; health services accessibility

Submitted Apr 15, 2018. Accepted for publication Apr 17, 2018.

doi: $10.21037 /$ tau.2018.04.20

View this article at: http://dx.doi.org/10.21037/tau.2018.04.20

\section{Introduction}

Access to care for men with infertility in the United States has been limited by a number of important barriers (1). Variation in the distribution of male infertility specialists and assisted reproductive technology (ART) centers throughout the country has led to disparities in access to care $(2,3)$. These geographic disparities have been exacerbated by economic disparities and limited insurance coverage, which forces many men to seek infertility care outside of traditional reimbursement models (4-6). 
Table 1 Infertility diagnosis among men who underwent semen analysis (SA)

\begin{tabular}{lcc}
\hline \multirow{2}{*}{ Patients } & \multicolumn{2}{c}{ Infertility diagnosis $(\%)$} \\
\cline { 2 - 3 } & Yes (N=359) & No (N=268) \\
\hline Normal SA & $128(35.7)$ & $211(78.7)$ \\
Abnormal SA & $78(21.7)$ & $57(21.3)$ \\
Oligospermia & $38(10.6)$ & $21(7.8)$ \\
Severe oligospermia & $26(7.2)$ & $15(5.6)$ \\
Azoospermia & $14(3.9)$ & $21(7.8)$ \\
No SA & $153(42.6)$ & $0(0)$ \\
\hline
\end{tabular}

Male military veterans sit at the confluence of these barriers and are among the most vulnerable populations for inadequate access to quality of care. These men have greater risk of infertility than the general population with an estimated $13.8 \%$ lifetime prevalence $(7,8)$. However, male military veterans are less likely to seek or receive infertility care than their female veteran counterparts (7). In order to address the unmet need of male veterans with infertility, an amendment to the Military Construction-Veterans Affairs (VA) Appropriations Bill, effective January 2017, temporarily extended coverage for ART to male veterans with infertility related to their military service (9). However, the effectiveness of this legislation is dependent upon both the quality of infertility care provided to male veterans and the eligibility criteria of the legislation-neither of which has been well characterized.

In order to assess the effectiveness of the new legislation in overcoming barriers to male infertility care, we first sought to characterize the appropriateness of infertility evaluation and diagnosis in men presenting to the local VA healthcare system. We further evaluated changes in the number of men seeking fertility work-up following the new legislation and characterized men who qualified for the newly sponsored in vitro fertilization (IVF) consultation and treatment.

\section{Methods}

We identified all male patients within the South Florida portion of the Veterans Integrated Service Networks (VISN) 8: VA Sunshine Healthcare Network who underwent a semen analysis (SA) or were assigned a diagnosis code of male infertility according to the International Classification of Diseases (ICD) (ICD-9 code 606 or ICD-10 code N46) in the local Computerized Patient Record System (CPRS) between January 1999 and June 2017. Those with a prior history of vasectomy or vasovasostomy were excluded from the study. The proportion of men diagnosed with infertility who underwent SA was determined, and men were classified according to the World Health Organization (WHO) criteria as having normal sperm concentration, oligospermia ( $<15$ million sperm $/ \mathrm{mL})$, severe oligospermia ( $<5$ million sperm $/ \mathrm{mL}$ ), or azoospermia on the most recent SA. The proportion of patients within each group carrying a categorically-entered physician-assigned infertility diagnosis was determined.

In order to assess the effect of the new legislation, we identified all male patients ages 29 through 49 who underwent SA in the period before (January through June 2016) and after (January through June 2017) the legislation enactment. The proportion of men with SA found to have oligospermia before and after the legislation were compared using chi-squared test. Oligospermic men presenting after the legislation were further analyzed according to the type of fertility specialist (urologist versus endocrinologist) performing the evaluation. Men who did not proceed to IVF treatment were characterized according to IVF eligibility according to the legislative requirement of documented military service connection to the infertility diagnosis.

The study was approved as a Quality Improvement Project by the Miami VA. All statistical analyses were performed using SAS v9.4 statistical software for Windows (SAS Institute Inc., Cary, NC, USA). Statistical significance was determined at $\mathrm{P}$ value $<0.05$.

\section{Results}

Among 474 men who had SA, 206 (43.5\%) were diagnosed with infertility and 268 (56.5\%) were not (Table 1). An additional 153 men were diagnosed with male infertility without ever undergoing SA. Of men diagnosed with infertility, 128 (62.1\%) had a normal SA and 78 (37.9\%) had an abnormal SA. Of men not diagnosed with infertility, $57(21.3 \%)$ had abnormal SA-21 (7.8\%) oligospermia, 15 (5.6\%) severe oligospermia, and 21 (7.8\%) azoospermia.

A total of 29 and 40 men underwent fertility workup before and after the legislation, respectively (Table 2). A greater number of men had oligospermia and severe oligospermia in the period after the legislation (1 versus 6 and 1 versus 10).

In the period following the legislation, 10 men presented 
Table 2 Sperm concentration and service-related component of infertility diagnosis among men presenting with oligospermia immediately before and after legislation

\begin{tabular}{lcc}
\hline Patients & Pre-legislation & Post-legislation \\
\hline Number of patients & 2 & 16 \\
Oligospermia & $1(50 \%)$ & $6(38 \%)$ \\
Severe oligospermia & $1(50 \%)$ & $10(63 \%)$ \\
Sperm concentration (million/mL) & $6.3(0.0-12.6)$ & $2.3(0.0-6.3)$ \\
Component of infertility diagnosis attributable to military service & & $8(50 \%)$ \\
$50-100 \%$ & $2(100 \%)$ & $3(19 \%)$ \\
$<50 \%$ & $0(0 \%)$ & $5(31 \%)$ \\
$0 \%$ & $0(0 \%)$ & \\
\hline
\end{tabular}

*, sperm concentration presented as median (interquartile range).

with severe oligospermia and were evaluated for IVF consultation eligibility. Among these patients, the infertility specialist was an urologist in $8(80 \%)$ of cases and an endocrinologist in $2(20 \%)$ of cases. Only 3 (30\%) of these veterans proceeded to IVF consultation, with $2(20 \%)$ eventually approved for ART. Of the 7 men who did not qualify for IVF consultation, all (100\%) were excluded due to lack of documented military service connection to the infertility diagnosis by the evaluating physician.

\section{Discussion}

As the largest single provider of healthcare to men in the United States, the policies and procedures of the VA have a crucial impact upon the ability to meet national demand for men's health services. In many domains of urologic care, the VA benefits package provides comprehensive coverage for both medical and procedural treatments. For example, sexual health coverage includes use of medical therapy such as phosphodiesterase-5 inhibitors, intracavernosal injections, and even penile prosthesis placement for refractory erectile dysfunction and Peyronie's disease (9). In contrast, coverage for male factor infertility has been historically limited. TRICARE, the managed care program for the Department of Defense Military Healthy System, provides coverage for diagnostic evaluation and therapeutic procedures for "physical causes of infertility" such as varicocelectomy, but not ART itself (10). The National Defense Authorization Act for 2008 allowed for provision of ART to certain active duty service members, but multiple subsequent attempts to expand coverage to military veterans have failed. Ultimately, after lobbying assistance from the American Medical
Association, the "Fertility Counseling and Treatment for Certain Veterans and Spouses" amendment of the MilConVA Appropriations bill became effective in January 2017, providing ART to a veteran or spouse meeting specific conditions, including an infertility diagnosis by a specified infertility provider and a documented service connection to the infertility diagnosis.

We found discordance between the evaluation and diagnosis of male infertility, which likely serves as an initial barrier to ART treatments for veterans. A substantial portion of men with abnormal SA were not assigned an infertility diagnosis. Conversely, a large number of men were diagnosed with infertility despite never undergoing SA or an SA with normal semen parameters. These findings suggest an inadequacy in the physicians responsible for referrals to infertility providers, the infertility providers, or the infertility evaluation itself. While the VA has recently published a directive defining the parameters of infertility evaluation and treatment, it does not include specific guidelines regarding who should be screened/ offered infertility evaluation or the mechanisms for doing so. More specific protocols for infertility evaluation, along with education of primary care providers and specialists throughout the VA system, may help to overcome this initial barrier to infertility care.

Only $30 \%$ of men with the diagnosis of infertility by a specialist proceeded to ART, while the remaining patients were disqualified due to lack of service connection. With half of the oligospermic men found to have a $<50 \%$ service-related etiology for their infertility, the service connection designation appears to be a substantial barrier to receiving ART. The designation of service connection 
may be made by any physician, who may not be familiar with the potential harmful effects of occupational exposures endured by veterans. Furthermore, the mechanism for designating service connection within the VA CPRS is complex and internally redundant. CPRS allows designation of a medical condition as either $50-100 \%,<50 \%$, or not at all connected to military service. In the case of male infertility, this designation is first assigned by a physician and later characterized by an infertility provider in the form of a simple "yes" or "no" question. We found discordance between these two designations in the majority of oligospermic men, which may further impair appropriate evaluation and treatment for these men.

Our results suggest that the recent legislation has not substantially increased access to ART for military veterans. Efforts to improve access within the confines of the current legislation should focus on the education of primary care providers and specialists to optimize the sensitivity and specificity of an infertility diagnosis within the VA system. Furthermore, an attempt to streamline the service connection designation, either through reform of the infertility evaluation or deferral of this role to the infertility provider alone, may increase access to infertility treatment and ART. Beyond these interventions, legislative change is likely necessary as the coverage provisions for the current legislation are temporary and set to expire in 2018. We hope that our results will help to inform the legislative process and ensure that the next iteration will maximize benefits and coverage for veterans.

Our study must be interpreted within the context of certain limitations. First, due to the catchment area and patient population of the South Florida VA system, the sample size of men with infertility was relatively small. Second, the recent nature of the legislation resulted in a short post-legislation study period, which may not fully capture the effects of the legislation, as there may be an adjustment period for administrators and providers to learn and implement the new policies. Third, we utilized both oligospermia and azoospermia as surrogates for male infertility. However, oligospermic men may not be infertile or subfertile; furthermore, it is not possible to discern whether these men were actively attempting to conceive and thus met the gold standard definition of infertility. Fourth, medical records for patients outside of the VA system were not available. As such, men with a diagnosis of infertility in the absence of SA may have had SA performed prior to presentation at the VA, thereby rendering this diagnosis appropriate.
In conclusion, among men presenting for fertility evaluation in the South Florida VA system, we found discordance between the evaluation and diagnosis of male infertility, which likely serves as an initial barrier to ART treatments for veterans. Despite legislation intended to increase access to ART, most veterans with a diagnosis of infertility did not proceed to ART treatments due to lack of service connection to the infertility diagnosis, which substantially curtails the effect of this legislation. Improvements in point of care infertility evaluation and diagnosis, along with the development of new legislation, may increase access to ART for military veterans with infertility.

\section{Acknowledgements}

Dr. Ramasamy acknowledges his employment as a contractor at the Bruce Carter VA Medical Center in Miami, FL.

\section{Footnote}

Conflicts of Interest: The authors have no conflicts of interest to declare.

Ethical Statement: The study was approved as a Quality Improvement Project by the Miami VA.

Disclaimer: The content presented herein is the responsibility of the authors alone and does not necessarily reflect the views or policies of the Department of Veterans Affairs or the United States Government.

\section{References}

1. Adashi EY, Dean LA. Access to and use of infertility services in the United States: framing the challenges. Fertil Steril 2016;105:1113-8.

2. Nangia AK, Likosky DS, Wang D. Distribution of male infertility specialists in relation to the male population and assisted reproductive technology centers in the United States. Fertil Steril 2010;94:599-609.

3. Nangia AK, Likosky DS, Wang D. Access to assisted reproductive technology centers in the United States. Fertil Steril 2010;93:745-61.

4. Dupree JM. Insurance coverage for male infertility care in the United States. Asian J Androl 2016;18:339-41.

5. Meacham RB, Joyce GF, Wise M, et al. Male infertility. J Urol 2007;177:2058-66. 
6. Dupree JM, Dickey RM, Lipshultz LI. Inequity between male and female coverage in state infertility laws. Fertil Steril 2016;105:1519-22.

7. Katon J, Cypel Y, Raza M, et al. Self-reported infertility among male and female veterans serving during Operation Enduring Freedom/Operation Iraqi Freedom. J Womens Health (Larchmt) 2014;23:175-83.

8. Maconochie N, Doyle P, Carson C. Infertility among male

Cite this article as: Halpern JA, Jue JS, Ramasamy R. Infertility evaluation and access to assisted reproductive technologies among male military veterans: analysis of the South Florida veterans affairs experience. Transl Androl Urol 2018;7(Suppl 2):S188-S192. doi: 10.21037/tau.2018.04.20
UK veterans of the 1990-1 Gulf war: reproductive cohort study. BMJ 2004;329:196-201.

9. Fenstermaker M, Paknikar S, Rambhatla A, et al. The State of Men's Health Services in the Veterans Health Administration. Curr Urol Rep 2017;18:88.

10. Lund PS. Infertility Benefits for Veterns. American Medical Association, 2016. 